SUPPORTING INFORMATION FOR:

\title{
Revealing the structural plasticity of SARS-CoV-2 nsp7 and nsp8 using structural proteomics
}

\author{
Valentine V. Courouble ${ }^{1, *}$, Sanjay Kumar Dey2,", Ruchi Yadav ${ }^{2,}$, Jennifer Timm², \\ Jerry Joe E. K. Harrison²,4, Francesc X. Ruiz², Eddy Arnold2,**, and Patrick R. Griffin 1, 3,**
}

${ }^{1}$ Department of Molecular Medicine, The Scripps Research Institute, Jupiter, FL 33458, USA

${ }^{2}$ Center for Advanced Biotechnology \& Medicine, and Department of Chemistry \& Chemical Biology, Rutgers University, Piscataway, NJ 08854, USA,

${ }^{3}$ Department of Integrative Structural and Computational Biology, The Scripps Research Institute, Jupiter, Florida 33458, USA,

${ }^{4}$ Department of Chemistry, School of Physical and Mathematical Sciences, University of Ghana, Legon, Box LG 56, Legon-Accra, Ghana

${ }^{*}$ Contributed equally

${ }^{* *}$ To whom correspondence should be addressed:

pgriffin@scripps.edu and arnold@cabm.rutgers.edu 
Table S1. nsp8 peptides sequences shown with their corresponding charge state and residue start and end number based on sequence. The perturbation values $\Delta \% \mathrm{D}$ reported with propagated errors are shown in parenthesis.

\begin{tabular}{|c|c|c|c|c|c|c|}
\hline nsp8 Sequence & Charge & Start & End & Features & $\begin{array}{c}\text { nsp8 vs } \\
\text { nsp7nsp8 } \\
1: 1\end{array}$ & $\begin{array}{c}\text { nsp8 vs } \\
\text { nsp7nsp8 } \\
3: 1\end{array}$ \\
\hline FSSLPSY & 1 & 6 & 12 & & $-1(3)$ & $1(2)$ \\
\hline FSSLPSYAAF & 2 & 6 & 15 & & $-1(3)$ & $0(2)$ \\
\hline ATAQEAYEQAVANGDS & 2 & 16 & 31 & & $4(2)$ & $0(2)$ \\
\hline ATAQEAYEQAVANGDSE & 2 & 16 & 32 & & $5(2)$ & $5(2)$ \\
\hline ATAQEAYEQAVANGDSEVVLKKLKKSLNVAKSEFDRDAAM & 4 & 16 & 55 & & $3(2)$ & $5(1)$ \\
\hline YEQAVANGDSE & 2 & 22 & 32 & & $-1(2)$ & $0(2)$ \\
\hline $\begin{array}{l}\text { YEQAVANGDSEVVLKKLKKSLNVAKSEFDRDAAM } \\
\end{array}$ & 4 & 22 & 55 & & $3(2)$ & $2(2)$ \\
\hline VVLKKLKKSLNVAKSEF & 2 & 33 & 49 & & $5(3)$ & $3(1)$ \\
\hline VVLKKLKKSLNVAKSEF & 3 & 33 & 49 & & $5(2)$ & $4(3)$ \\
\hline QRKLEKMAD & 2 & 56 & 64 & & $1(3)$ & $4(3)$ \\
\hline QRKLEKMAD & 3 & 56 & 64 & & $-1(3)$ & $4(3)$ \\
\hline QRKLEKMADQAM & 3 & 56 & 67 & & $0(3)$ & $1(3)$ \\
\hline QRKLEKMADQAMTQM & 2 & 56 & 70 & & $-2(3)$ & $-2(3)$ \\
\hline QRKLEKMADQAMTQM & 3 & 56 & 70 & & $0(3)$ & $1(3)$ \\
\hline QRKLEKMADQAMTQM & 4 & 56 & 70 & & $0(3)$ & $0(3)$ \\
\hline YKQARSEDKRAKVTSAMQTML & 2 & 71 & 91 & [H1:14] & $-2(2)$ & $-7(2)$ \\
\hline YKQARSEDKRAKVTSAMQTML & 3 & 71 & 91 & {$[\mathrm{H} 1: 14]$} & $-1(3)$ & $-6(2)$ \\
\hline YKQARSEDKRAKVTSAMQTML & 4 & 71 & 91 & {$[\mathrm{H} 1: 14]$} & $-1(3)$ & $-6(2)$ \\
\hline LRKLDNDAL & 3 & 95 & 103 & {$[\mathrm{H} 1: 5 \mathrm{H} 2: 2]$} & $-3(2)$ & $-11(3)$ \\
\hline LRKLDNDALNNIIINNARDGCVPLNIIPLTT & 4 & 95 & 124 & [H1:5 H2:12] & $-7(4)$ & $-13(4)$ \\
\hline RKLDNDALNNIINNARDGCVPLNIIPLTT & 3 & 96 & 124 & [H1:4 H2:12] & $-6(2)$ & $-15(3)$ \\
\hline RKLDNDALNNIINNARDGCVPLNIIPLTT & 4 & 96 & 124 & [H1:4 H2:12] & $-6(3)$ & $-14(3)$ \\
\hline RKLDNDALNNIINNARDGCVPLNIIPLTTA & 4 & 96 & 125 & [H1:4 H2:12] & $-6(2)$ & $-9(2)$ \\
\hline RKLDNDALNNIIINNARDGCVPLNIIPLTTAAKL & 4 & 96 & 128 & [H1:4 H2:12 B1:3] & $-6(3)$ & $-10(2)$ \\
\hline NNIINNARDGCVPLNIIPLTT & 2 & 104 & 124 & {$[\mathrm{H} 2: 10]$} & $-7(3)$ & $-13(3)$ \\
\hline NNIINNARDGCVPLNIIPLTTA & 2 & 104 & 125 & {$[\mathrm{H} 2: 10]$} & $-9(3)$ & $-14(3)$ \\
\hline NNIINNARDGCVPLNIIPLTTAAKL & 3 & 104 & 128 & [H2:10 B1:3] & $-6(2)$ & $-8(2)$ \\
\hline MVVIPDYNT & 1 & 129 & 137 & [B1:5 H3:2] & $2(3)$ & $-7(2)$ \\
\hline MVVIPDYNTYKNTCDGTTF & 2 & 129 & 147 & [B1:5 H3:7 B2:1] & $3(3)$ & $-7(2)$ \\
\hline MVVIPDYNTYKNTCDGTTFT & 2 & 129 & 148 & [B1:5 H3:7 B2:2] & $2(2)$ & $-7(2)$ \\
\hline YASAL & 1 & 149 & 153 & [B2:2 B3:1] & $-9(4)$ & $-10(3)$ \\
\hline WEIQQVVDADSKIVQL & 2 & 154 & 169 & [B3:8] & $4(2)$ & $-2(3)$ \\
\hline DSKIVQL & 1 & 163 & 169 & & $2(2)$ & $-2(3)$ \\
\hline DSKIVQL & 2 & 163 & 169 & & $2(2)$ & $-2(3)$ \\
\hline SEISM & 1 & 170 & 174 & & $0(3)$ & $2(3)$ \\
\hline SEISMDNSPNLAWPLIVTAL & 2 & 170 & 189 & [B4:5] & $2(1)$ & $-1(1)$ \\
\hline ISMDNSPNLAWPLIVTAL & 2 & 172 & 189 & [B4:5] & $2(1)$ & $-2(1)$ \\
\hline DNSPNLAWPLIVT & 2 & 175 & 187 & [B4:3] & $5(1)$ & $-3(1)$ \\
\hline DNSPNLAWPLIVTA & 2 & 175 & 188 & [B4:4] & $4(1)$ & $0(2)$ \\
\hline DNSPNLAWPLIVTAL & 2 & 175 & 189 & [B4:5] & $1(1)$ & $1(1)$ \\
\hline LRANSA & 2 & 189 & 194 & [B4:3] & $-2(4)$ & $2(3)$ \\
\hline RANSAVKLQ & 2 & 190 & 198 & {$[\mathrm{~B} 4: 2]$} & $2(4)$ & $1(3)$ \\
\hline QENLYFQ & 2 & 198 & 204 & [Tag:6] & $-3(2)$ & $4(2)$ \\
\hline
\end{tabular}


Table S2. nsp7 peptides sequences shown with their corresponding charge state and residue start and end number based on sequence. The perturbation values $\Delta \% \mathrm{D}$ reported with propagated errors are shown in parenthesis.

\begin{tabular}{|c|c|c|c|c|c|c|}
\hline nsp7 Sequence & Charge & Start & End & Features & $\begin{array}{c}\text { nsp7 vs } \\
\text { nsp7nsp8 } \\
1: 1\end{array}$ & $\begin{array}{c}\text { nsp7 vs } \\
\text { nsp7nsp8 } \\
3: 1\end{array}$ \\
\hline SKMSDVKC & 2 & 1 & 8 & [H1:6] & $-2(3)$ & $-20(3)$ \\
\hline SKMSDVKCTS & 1 & 1 & 10 & [H1:8] & $-2(3)$ & $-15(3)$ \\
\hline SKMSDVKCTS & 2 & 1 & 10 & [H1:8] & $-2(4)$ & $-15(3)$ \\
\hline SKMSDVKCTS & 3 & 1 & 10 & {$[\mathrm{H} 1: 8]$} & $-2(4)$ & $-16(3)$ \\
\hline SKMSDVKCTSV & 2 & 1 & 11 & [H1:9] & $-3(4)$ & $-16(3)$ \\
\hline SKMSDVKCTSVVL & 2 & 1 & 13 & [H1:11] & $-4(4)$ & $-14(5)$ \\
\hline SKMSDVKCTSVVL & 3 & 1 & 13 & [H1:11] & $-4(4)$ & $-17(4)$ \\
\hline VLLSVL & 1 & 12 & 17 & {$[\mathrm{H} 1: 6]$} & $-3(3)$ & $2(4)$ \\
\hline LSVLQQ & 1 & 14 & 19 & [H1:6] & $2(2)$ & $3(4)$ \\
\hline LSVLQQL & 2 & 14 & 20 & {$[\mathrm{H} 1: 7]$} & $0(2)$ & $1(2)$ \\
\hline QQLRVESSSKL & 2 & 18 & 28 & [H1:4 H2:2] & $0(3)$ & $-7(3)$ \\
\hline QQLRVESSSKL & 3 & 18 & 28 & [H1:4 H2:2] & $0(4)$ & $-8(3)$ \\
\hline LRVESSSKL & 2 & 20 & 28 & [H1:2 H2:2] & $-2(4)$ & $-14(2)$ \\
\hline RVESSSKL & 2 & 21 & 28 & [H1:1 H2:2] & $-2(4)$ & $-17(2)$ \\
\hline VQLHNDIL & 1 & 33 & 40 & {$[\mathrm{H} 2: 8]$} & $2(2)$ & $4(2)$ \\
\hline VQLHNDIL & 2 & 33 & 40 & {$[\mathrm{H} 2: 8]$} & $1(2)$ & $4(2)$ \\
\hline VQLHNDILL & 2 & 33 & 41 & [H2:9] & $3(2)$ & $4(2)$ \\
\hline HNDILL & 1 & 36 & 41 & {$[\mathrm{H} 2: 6]$} & $3(3)$ & $5(3)$ \\
\hline LAKDTTEA & 2 & 41 & 48 & {$[\mathrm{H} 2: 2 \mathrm{H} 3: 3]$} & $-1(4)$ & $-8(2)$ \\
\hline LAKDTTEAF & 2 & 41 & 49 & {$[\mathrm{H} 2: 2 \mathrm{H3}: 4]$} & $-1(3)$ & $-8(3)$ \\
\hline AKDTTEAF & 2 & 42 & 49 & {$[\mathrm{H} 2: 1 \mathrm{H} 3: 4]$} & $-1(4)$ & $-7(4)$ \\
\hline FEKMVSL & 1 & 49 & 55 & [H3:7] & $0(3)$ & $5(3)$ \\
\hline FEKMVSL & 2 & 49 & 55 & [H3:7] & $1(3)$ & $4(3)$ \\
\hline FEKMVSLL & 1 & 49 & 56 & [H3:8] & $0(2)$ & $3(2)$ \\
\hline FEKMVSLL & 2 & 49 & 56 & {$[\mathrm{H} 3: 8]$} & $0(2)$ & $3(2)$ \\
\hline LSMQGAVDINKL & 1 & 60 & 71 & [H3:3] & $0(3)$ & $-7(2)$ \\
\hline LSMQGAVDINKLCE & 2 & 60 & 73 & [H3:3] & $-2(2)$ & $-8(3)$ \\
\hline LSMQGAVDINKLCEE & 2 & 60 & 74 & [H3:3] & $-2(3)$ & $-9(3)$ \\
\hline AVDINKL & 2 & 65 & 71 & & $-2(3)$ & $-5(3)$ \\
\hline AVDINKLCE & 1 & 65 & 73 & & $-2(2)$ & $-4(3)$ \\
\hline AVDINKLCE & 2 & 65 & 73 & {$[\mathrm{H} 4: 7]$} & $-2(2)$ & $-4(3)$ \\
\hline EMLDNRATL & 2 & 74 & 82 & {$[\mathrm{H} 4: 6]$} & $-2(3)$ & $-13(3)$ \\
\hline EMLDNRATLQENL & 2 & 74 & 86 & [H4:6 Tag:2] & $-1(4)$ & $-11(3)$ \\
\hline MLDNRATL & 2 & 75 & 82 & {$[\mathrm{H} 4: 5]$} & $-2(4)$ & $-18(2)$ \\
\hline MLDNRATLQE & 2 & 75 & 84 & [H4:5] & $-3(4)$ & $-17(2)$ \\
\hline MLDNRATLQENL & 2 & 75 & 86 & [H4:5 Tag:2] & $-4(5)$ & $-14(3)$ \\
\hline LDNRATL & 2 & 76 & 82 & {$[\mathrm{H} 4: 4]$} & $-3(4)$ & $-18(3)$ \\
\hline LDNRATLQENL & 2 & 76 & 86 & [H4:4 Tag:2] & $-1(4)$ & $-15(3)$ \\
\hline QENLYFQ & 1 & 83 & 89 & [Tag:5] & $-3(3)$ & $-3(2)$ \\
\hline
\end{tabular}


A $\quad$ nsp7 v nsp7:nsp8 (1:1)

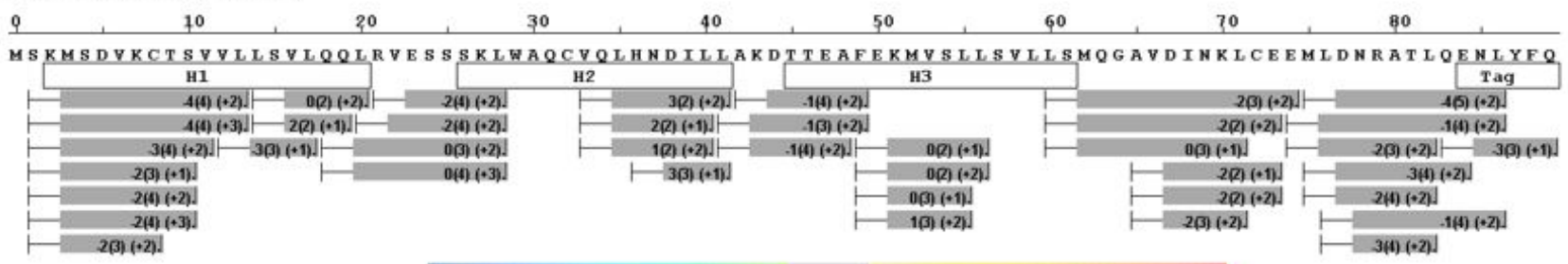

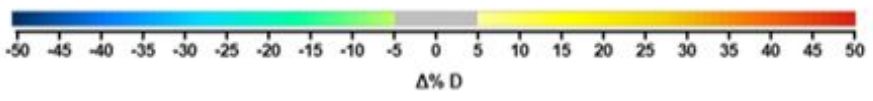

B $\quad$ nsp8 v nsp7:nsp8 (1:1)

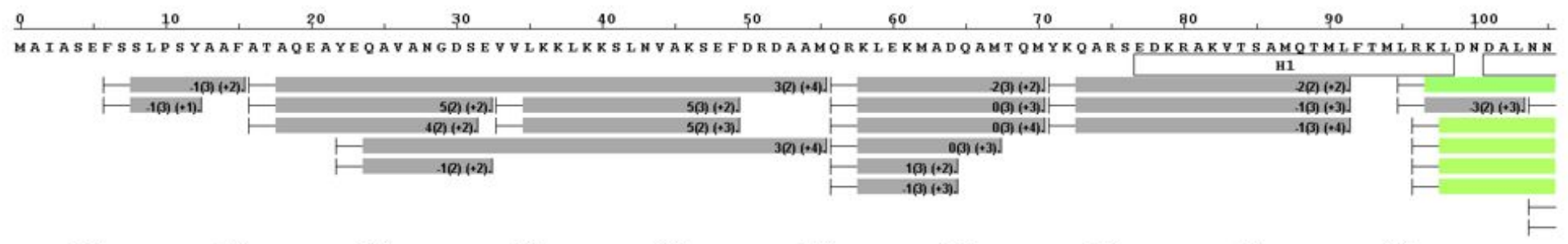

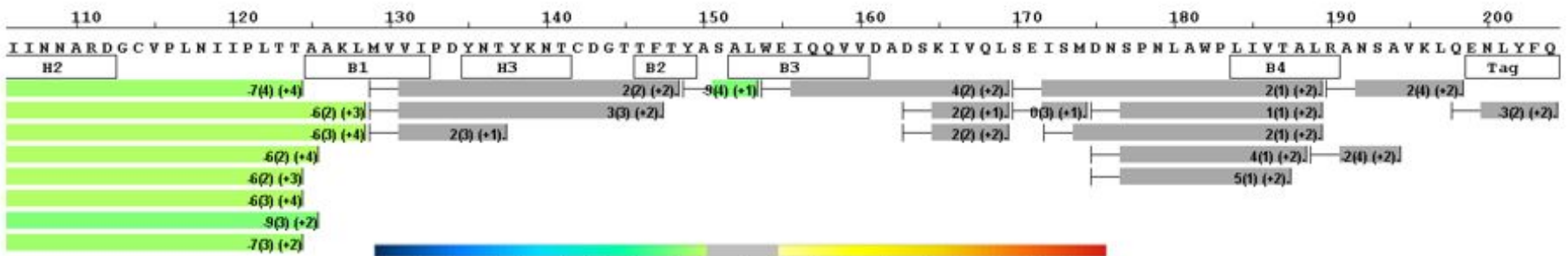

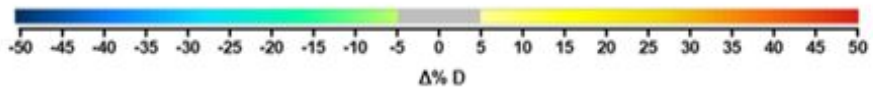

C nsp7 v nsp7:nsp8 (1:3)
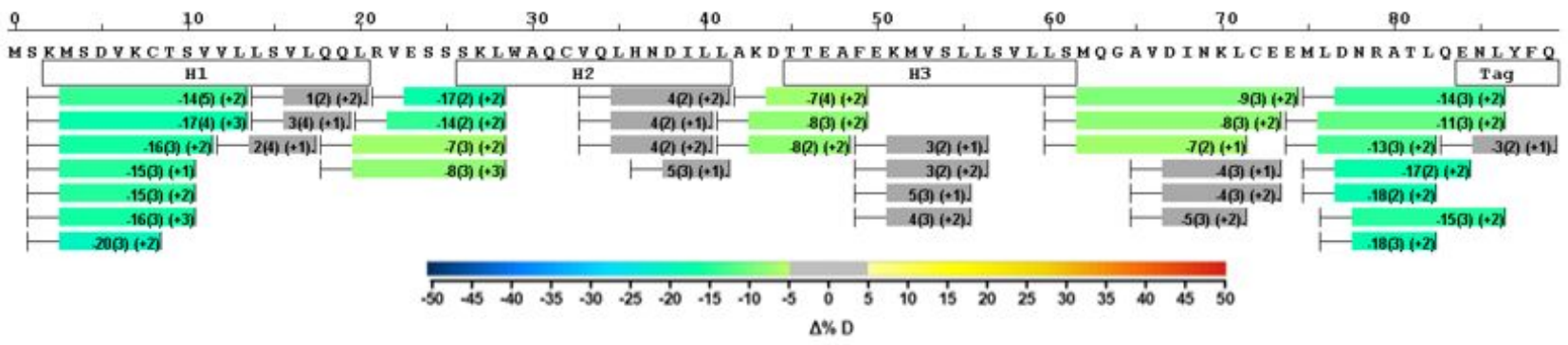

D $\quad$ nsp8 v nsp7:nsp8 (3:1)
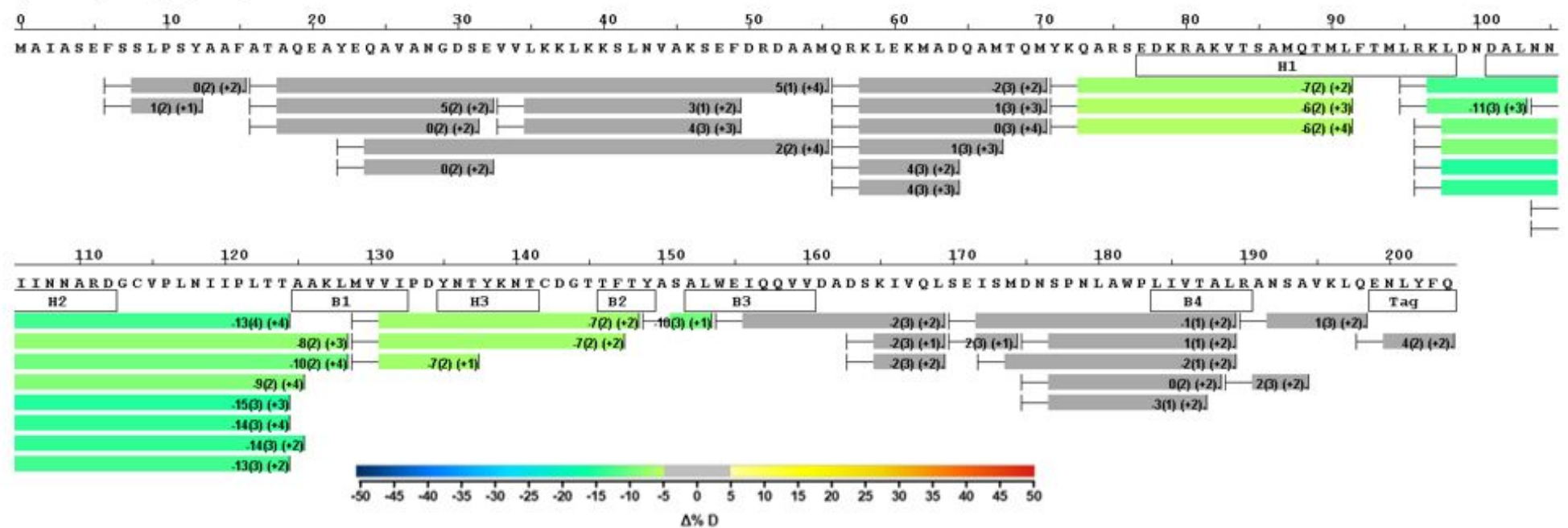

Figure S1. Sequence coverage for differential HDX-MS analysis of nsp7 vs nsp7:nsp8 1:1 (A), nsp8 vs nsp7:nsp8 1:1 (B), nsp7 vs nsp7:nsp8 1:3 (C), and nsp8 vs nsp7:nsp8 3:1 (D). 

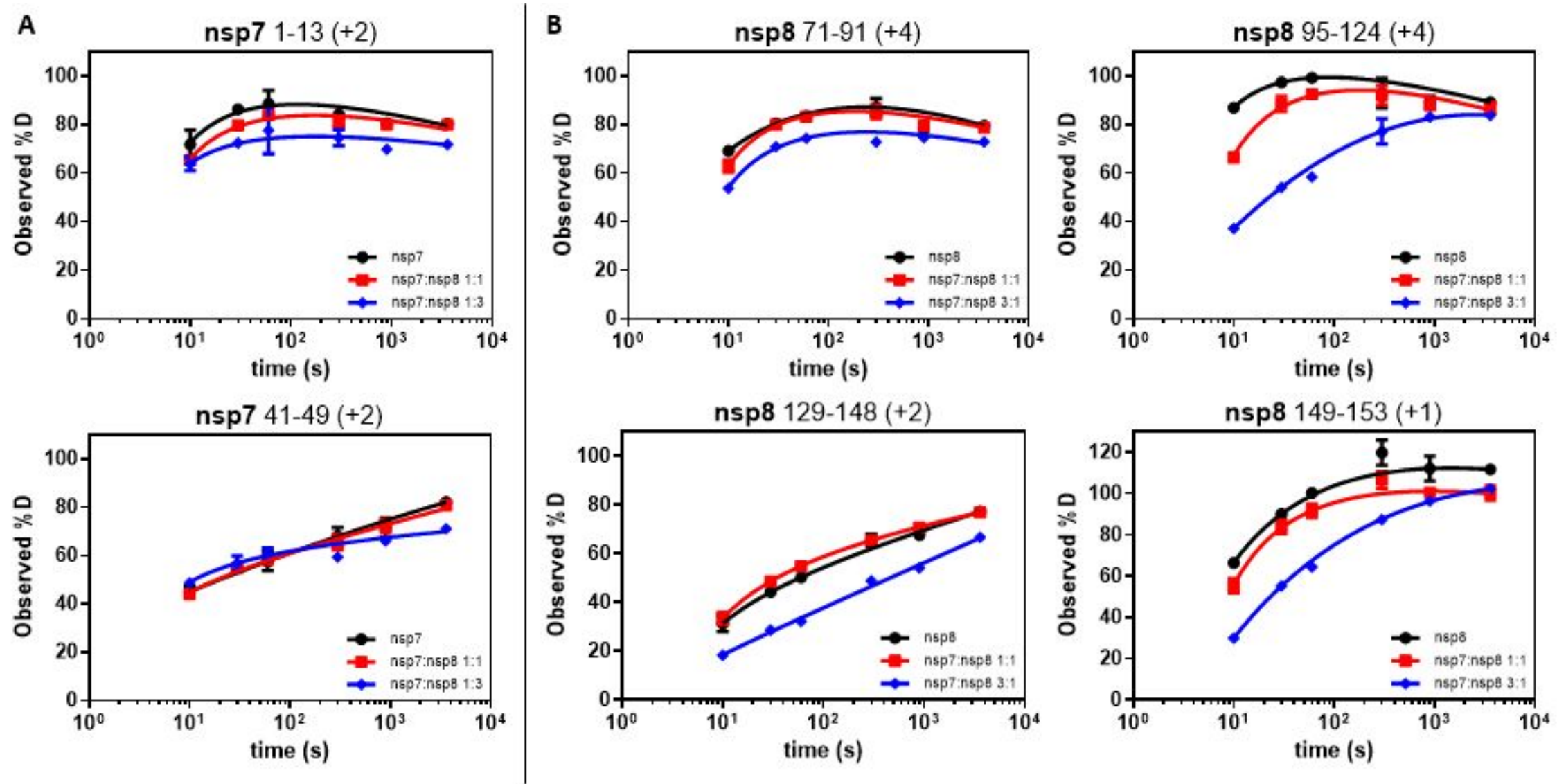

Figure S2. Representative deuterium build-up plots showing regions of protection in nsp7 (A) and nsp8 (B) upon complex formation. 
Table S3. HDX-MS experimental conditions and data analysis parameters from the guidelines of the IC-HDX-MS community

\begin{tabular}{|c|c|c|}
\hline Data Set (Figure 2B, S1A) & nsp7 & nsp7:nsp8 1:1 \\
\hline HDX reaction details & $\begin{array}{c}50 \mathrm{mM} \text { HEPES, } 500 \mathrm{mM} \mathrm{NaCl}, \\
1 \mathrm{mM} \text { TCEP, } \mathrm{pH}=8.0,30 \mathrm{~min}, \\
25^{\circ} \mathrm{C}\end{array}$ & $\begin{array}{c}50 \mathrm{mM} \text { HEPES, } 500 \mathrm{mM} \mathrm{NaCl} \text {, } \\
1 \mathrm{mM} \text { TCEP, } \mathrm{pH}=8.0,30 \mathrm{~min}, \\
25^{\circ} \mathrm{C}\end{array}$ \\
\hline HDX quench reaction details & $\begin{array}{c}5 \mathrm{M} \text { Urea, } 1 \% \mathrm{TFA}, \mathrm{pH}=2.0 \\
4{ }^{\circ} \mathrm{C}\end{array}$ & $\begin{array}{c}5 \mathrm{M} \text { Urea, } 1 \% \mathrm{TFA}, \mathrm{pH}=2.0 \\
4{ }^{\circ} \mathrm{C}\end{array}$ \\
\hline HDX time course (sec) & $10,30,60,900,3600$ & $10,30,60,900,3600$ \\
\hline Back-exchange & \multicolumn{2}{|c|}{$\begin{array}{l}\text { estimated from input recovery estimate of } 0.7 \text { and } \\
\text { deuterium solution concentration of } 0.8\end{array}$} \\
\hline \# of Peptides & \multicolumn{2}{|c|}{94} \\
\hline Sequence coverage & \multicolumn{2}{|c|}{$99 \%$} \\
\hline $\begin{array}{l}\text { Average peptide length / } \\
\text { Redundancy }\end{array}$ & \multicolumn{2}{|c|}{$12 / 12.494$} \\
\hline Replicates (biological or technical) & \multicolumn{2}{|c|}{3 (technical) } \\
\hline Repeatability & \multicolumn{2}{|c|}{3.17193 (average STD) } \\
\hline Significant differences in HDX & \multicolumn{2}{|c|}{$>5 \% \mathrm{D}$ (unpaired t-tests at each time point, $\mathrm{p}$-value $<0.01$ ) } \\
\hline Data Set (Figure 2D, S1C) & \multicolumn{2}{|r|}{ nsp7:nsp8 1:3 } \\
\hline HDX reaction details & $\begin{array}{c}50 \mathrm{mM} \text { HEPES, } 500 \mathrm{mM} \mathrm{NaCl}, \\
1 \mathrm{mM} \text { TCEP, } \mathrm{pH}=8.0,30 \mathrm{~min}, \\
25^{\circ} \mathrm{C}\end{array}$ & $\begin{array}{c}50 \mathrm{mM} \text { HEPES, } 500 \mathrm{mM} \mathrm{NaCl} \text {, } \\
1 \mathrm{mM} \text { TCEP, } \mathrm{pH}=8.0,30 \mathrm{~min}, \\
25^{\circ} \mathrm{C}\end{array}$ \\
\hline HDX quench reaction details & $\begin{array}{c}5 \mathrm{M} \text { Urea, } 1 \% \mathrm{TFA}, \mathrm{pH}=2.0 \\
4{ }^{\circ} \mathrm{C}\end{array}$ & $\begin{array}{c}5 \mathrm{M} \text { Urea, } 1 \% \mathrm{TFA}, \mathrm{pH}=2.0, \\
4{ }^{\circ} \mathrm{C}\end{array}$ \\
\hline HDX time course (sec) & $10,30,60,900,3600$ & $10,30,60,900,3600$ \\
\hline Back-exchange & \multicolumn{2}{|c|}{$\begin{array}{l}\text { estimated from input recovery estimate of } 0.7 \text { and } \\
\text { deuterium solution concentration of } 0.8\end{array}$} \\
\hline \# of Peptides & \multicolumn{2}{|c|}{94} \\
\hline Sequence coverage & \multicolumn{2}{|c|}{$99 \%$} \\
\hline $\begin{array}{l}\text { Average peptide length / } \\
\text { Redundancy }\end{array}$ & \multicolumn{2}{|c|}{$12 / 12.494$} \\
\hline Replicates (biological or technical) & \multicolumn{2}{|c|}{3 (technical) } \\
\hline Repeatability & \multicolumn{2}{|c|}{2.904302 (average STD) } \\
\hline Significant differences in HDX & \multicolumn{2}{|c|}{$>5 \% \mathrm{D}$ (unpaired t-tests at each time point, $p$-value $<0.01$ ) } \\
\hline Data Set (Figure 2A, S1B) & \multicolumn{2}{|r|}{ nsp7:nsp8 1:1 } \\
\hline HDX reaction details & $\begin{array}{c}50 \mathrm{mM} \text { HEPES, } 500 \mathrm{mM} \mathrm{NaCl}, \\
1 \mathrm{mM} \text { TCEP, } \mathrm{pH}=8.0,30 \mathrm{~min}, \\
25^{\circ} \mathrm{C}\end{array}$ & $\begin{array}{c}50 \mathrm{mM} \text { HEPES, } 500 \mathrm{mM} \mathrm{NaCl} \text {, } \\
1 \mathrm{mM} \text { TCEP, } \mathrm{pH}=8.0,30 \mathrm{~min}, \\
25^{\circ} \mathrm{C}\end{array}$ \\
\hline HDX quench reaction details & $\begin{array}{l}5 \mathrm{M} \text { Urea, } 1 \% \mathrm{TFA}, \mathrm{pH}=2.0 \\
4{ }^{\circ} \mathrm{C}\end{array}$ & $\begin{array}{l}5 \mathrm{M} \text { Urea, } 1 \% \mathrm{TFA}, \mathrm{pH}=2.0 \\
4{ }^{\circ} \mathrm{C}\end{array}$ \\
\hline HDX time course (sec) & $10,30,60,900,3600$ & $10,30,60,900,3600$ \\
\hline Back-exchange & \multicolumn{2}{|c|}{$\begin{array}{c}\text { estimated from input recovery estimate of } 0.7 \text { and } \\
\text { deuterium solution concentration of } 0.8\end{array}$} \\
\hline
\end{tabular}




\begin{tabular}{|c|c|c|}
\hline \# of Peptides & \multicolumn{2}{|c|}{113} \\
\hline Sequence coverage & \multicolumn{2}{|c|}{$100 \%$} \\
\hline $\begin{array}{l}\text { Average peptide length / } \\
\text { Redundancy }\end{array}$ & \multicolumn{2}{|c|}{16 / 9.069} \\
\hline Replicates (biological or technical) & \multicolumn{2}{|c|}{3 (technical) } \\
\hline Repeatability & \multicolumn{2}{|c|}{2.611554 (average STD) } \\
\hline Significant differences in HDX & \multicolumn{2}{|c|}{$>5 \% \mathrm{D}$ (unpaired t-tests at each time point, $\mathrm{p}$-value $<0.01$ ) } \\
\hline Data Set (Figure 2C, S1D) & \multicolumn{2}{|c|}{ nsp8 $\quad$ nsp7:nsp8 3:1 } \\
\hline HDX reaction details & $\begin{array}{c}50 \mathrm{mM} \text { HEPES, } 500 \mathrm{mM} \mathrm{NaCl}, \\
1 \mathrm{mM} \text { TCEP, } \mathrm{pH}=8.0,30 \mathrm{~min}, \\
25^{\circ} \mathrm{C}\end{array}$ & $\begin{array}{c}50 \mathrm{mM} \text { HEPES, } 500 \mathrm{mM} \mathrm{NaCl} \text {, } \\
1 \mathrm{mM} \text { TCEP, } \mathrm{pH}=8.0,30 \mathrm{~min} \text {, } \\
25^{\circ} \mathrm{C}\end{array}$ \\
\hline HDX quench reaction details & $\begin{array}{c}5 \mathrm{M} \text { Urea, } 1 \% \mathrm{TFA}, \mathrm{pH}=2.0 \\
4{ }^{\circ} \mathrm{C}\end{array}$ & $\begin{array}{c}5 \mathrm{M} \text { Urea, } 1 \% \mathrm{TFA}, \mathrm{pH}=2.0, \\
4{ }^{\circ} \mathrm{C}\end{array}$ \\
\hline HDX time course $(\mathrm{sec})$ & $10,30,60,900,3600$ & $10,30,60,900,3600$ \\
\hline Back-exchange & \multicolumn{2}{|c|}{$\begin{array}{l}\text { estimated from input recovery estimate of } 0.7 \text { and } \\
\text { deuterium solution concentration of } 0.8\end{array}$} \\
\hline \# of Peptides & \multicolumn{2}{|c|}{113} \\
\hline Sequence coverage & \multicolumn{2}{|c|}{$99 \%$} \\
\hline $\begin{array}{l}\text { Average peptide length / } \\
\text { Redundancy }\end{array}$ & \multicolumn{2}{|c|}{$16 / 9.069$} \\
\hline Replicates (biological or technical) & \multicolumn{2}{|c|}{3 (technical) } \\
\hline Repeatability & \multicolumn{2}{|c|}{2.3636 (average STD) } \\
\hline Significant differences in HDX & \multicolumn{2}{|c|}{$>5 \% \mathrm{D}$ (unpaired t-tests at each time point, $\mathrm{p}$-value $<0.01$ ) } \\
\hline
\end{tabular}




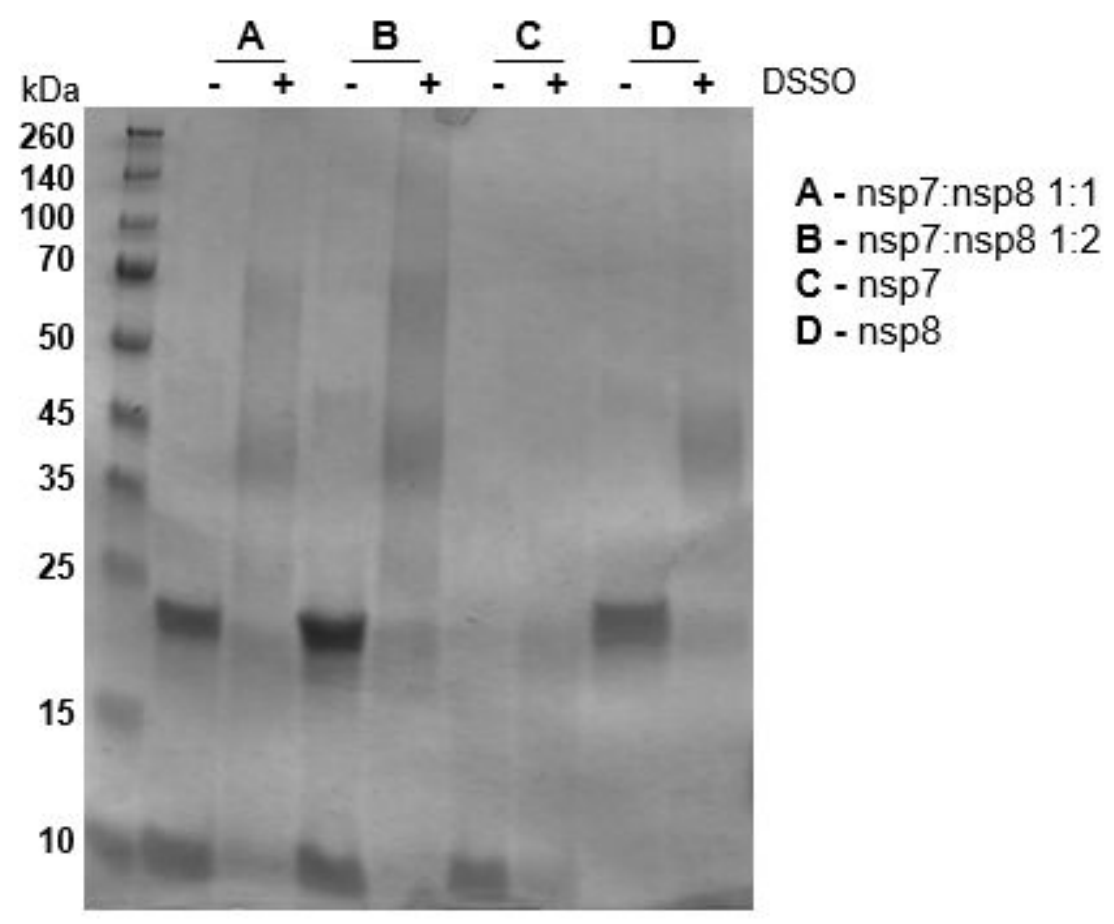

Figure S3. Crosslinking efficiency confirmed by SDS-PAGE. Presence of higher molecular weight bands and smeared bands in the sample with DSSO indicate successful crosslinking. 
A

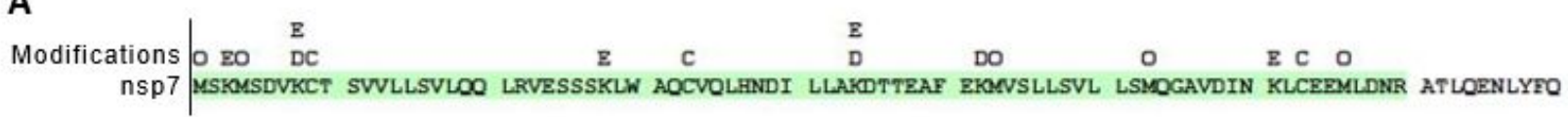

\section{B}

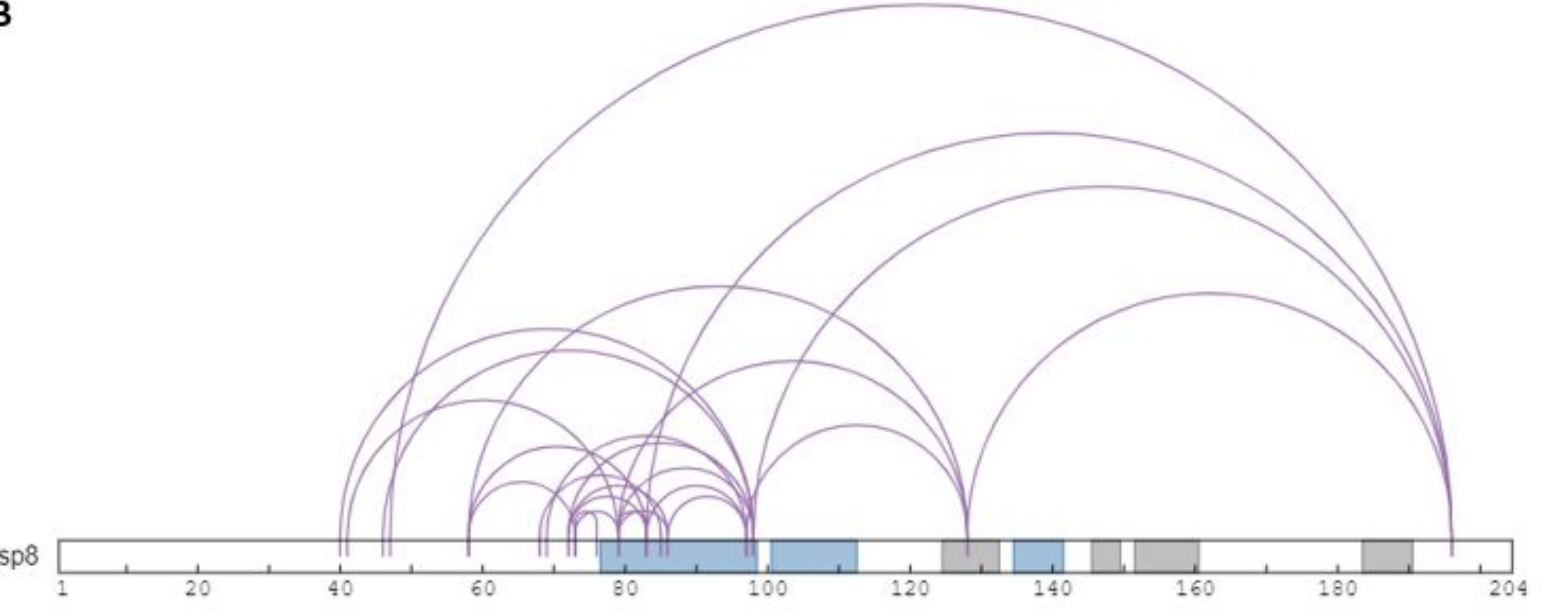

Figure S4. XL-MS of $n s p 7$ and nsp8 XL in isolation. (A) Sequence coverage of nsp7 XL-MS with modifications annotated - oxidation (O), carbamidomethyl (C), DSSO Tris (D), DSSO hydrolyzed (E), and N-terminal acetylation (A). (B) nsp8 crosslinks mapped onto nsp8 sequence with secondary structure from PDB:6YHU annotated with $\alpha$-helices in blue and $\beta$-sheets in gray. Initial Met residue removed from nsp8 sequence to maintain correct residue numbering. 
Table S4. Distances of inter-nsp7 nsp8 crosslinks mapped to all available SARS-CoV-2 nsp7:nsp8 structures. Columns color coded by grouping of structures based on conformation: dimer (green), cubic heterotetramer (yellow), linear heterotetramer (gray). Measure crosslink distances reported in $\AA$ and labeled in red if they violate the $26 \AA$ limit or in blue if they are below the $26 \AA$ limit.

\begin{tabular}{|c|c|c|c|c|c|c|c|c|c|c|c|c|c|c|c|c|c|c|c|c|c|c|c|}
\hline \multirow{3}{*}{ nsp7 } & \multirow{3}{*}{ nsp8 } & \multicolumn{12}{|c|}{ Dimer Interface } & \multicolumn{10}{|c|}{ Heterotetramer Interface } \\
\hline & & \multirow{2}{*}{$\begin{array}{c}\text { 6WIQ } \\
\text { (Å) }\end{array}$} & \multirow{2}{*}{$\begin{array}{l}\text { 6M5I } \\
\text { (Å) }\end{array}$} & \multicolumn{2}{|c|}{ 6WTC } & \multicolumn{2}{|c|}{ 6XIP } & \multicolumn{2}{|c|}{ 6WQD } & \multicolumn{2}{|c|}{ 7JLT } & \multicolumn{2}{|c|}{$6 \mathrm{YHU}$} & \multicolumn{2}{|c|}{ 6WTC } & \multicolumn{2}{|c|}{ 6XIP } & \multicolumn{2}{|c|}{ 6WQD } & \multicolumn{2}{|c|}{ 7JLT } & \multicolumn{2}{|c|}{ 6YHU } \\
\hline & & & & (Å) & (Å) & (Å) & (Å) & (Å) & (Å) & (Å) & (Å) & (Å) & (Å) & (Å) & (Å) & (Å) & (Å) & (Å) & (Å) & (Å) & (Å) & (Å) & (Å) \\
\hline 27 & 79 & 29.6 & 28.7 & 29.4 & 30.2 & 29.2 & 30.1 & 30.5 & 30.0 & 28.6 & 28.9 & 29.3 & 29.8 & 25.4 & 36.7 & 25.3 & 36.8 & 19.4 & 18.4 & 19.1 & 19.5 & 19.3 & 19.4 \\
\hline 27 & 196 & NF & $\mathrm{NF}$ & NF & $\mathrm{NF}$ & NF & NF & NF & 33.7 & $\mathrm{NF}$ & $\mathrm{NF}$ & $\mathrm{NF}$ & $\mathrm{NF}$ & NF & $\mathrm{NF}$ & $\mathrm{NF}$ & $\mathrm{NF}$ & $\mathrm{NF}$ & 61.1 & $\mathrm{NF}$ & $\mathrm{NF}$ & $\mathrm{NF}$ & NF \\
\hline 27 & 127 & 17.6 & 17.6 & 17.9 & 16.2 & 18.2 & 16.6 & 18.7 & 18.3 & 17.5 & 17.6 & 16.9 & 17.6 & 39.7 & 52.1 & 39.7 & 52.5 & 47.7 & 47.5 & 45.9 & 45.9 & 46.7 & 46.1 \\
\hline 70 & 79 & 18.9 & 18.7 & 19.2 & 18.9 & 18.5 & 18.5 & 18.9 & 19.0 & 18.4 & 18.8 & 18.5 & 18.1 & 43.7 & 25.7 & 43.0 & 25.7 & 34.6 & 33.6 & 37.5 & 38.2 & 34.4 & 33.9 \\
\hline 7 & 79 & 27.8 & 27.7 & 27.3 & 27.1 & 27.1 & 26.8 & 27.6 & 26.8 & 27.6 & 27.5 & 26.8 & 26.7 & 47.2 & 31.1 & 47.0 & 31.2 & 17.2 & 16.3 & 15.8 & 16.1 & 16.1 & 16.4 \\
\hline 43 & 79 & 38.7 & 38.5 & 38.4 & 37.7 & 38.3 & 37.3 & 38.3 & 37.9 & 38.2 & 38.4 & 37.3 & 37.5 & 50.5 & 32.6 & 50.1 & 32.7 & 15.6 & 15.3 & 14.2 & 14.7 & 14.5 & 14.9 \\
\hline 57 & 97 & 18.1 & 18.0 & 18.0 & 17.9 & 18.0 & 17.9 & 17.9 & 17.9 & 17.9 & 17.8 & 17.9 & 17.9 & 21.5 & 43.9 & 21.8 & 44.1 & 28.3 & 28.6 & 28.6 & 28.2 & 28.4 & 28.6 \\
\hline 7 & 97 & 13.4 & 13.2 & 13.0 & 13.1 & 13.1 & 13.0 & 12.8 & 13.0 & 13.2 & 13.5 & 13.0 & 12.8 & 34.3 & 45.8 & 34.5 & 45.9 & 13.7 & 14.0 & 14.9 & 14.7 & 14.1 & 14.0 \\
\hline 2 & 97 & 10.5 & 10.0 & 9.5 & 9.0 & 9.4 & 9.5 & 9.4 & 9.1 & 9.9 & 10.0 & 9.4 & 9.1 & 39.0 & 39.1 & 39.0 & 39.4 & 11.4 & 11.3 & 12.7 & 13.0 & 11.5 & 11.3 \\
\hline
\end{tabular}

